\title{
A simple device for the preparation of exactly aligned dichotic tapes
}

\author{
TERRY VINCENT and JOHN BRADSHAW \\ Monash University', Clay'ton, Victoria 3168, Australia
}

\begin{abstract}
A system is described involving three tape recorders and a control device whereby dichotic tapes can be prepared with onset alignment accurate to a few milliseconds, or to a measured asynchrony if required. No modifications of any sort are necessary for the three tape recorders. The two channels are separately recorded in the speaker's own time, the only requirement being that the separate words be spoken with an interword interval of $350 \mathrm{msec}$ or more, to provide sufficient time for starting and stopping the system. A tone burst is placed directly on tape on another channel ahead of each word, the latter being delayed by recording in tandem through two tape recorders. The first recorder is set to the delay (echo, NAB) facility, while the tone is recorded directly on the second. Subsequently, when this is done for each item on the two speech channels, the device, described below. utilizes the tone bursts to align the two input recorders. It then rerecords each pair of words on to the third (output or dichotic) machine, to the desired synchrony/asynchrony, and interitem interval. The system can be left to align the two channels automatically. A maximum repetition rate of two dichotic pairs every 1.3 sec can be achieved with Revox A77 tape recorders.
\end{abstract}

The dichotic presentation of stimuli, after the manner pioneered by Broadbent (1954) (usually three or more pairs of items are presented at a rate of about two pairs a second), is useful in investigating a number of problems, for example, selective attention (Lewis, 1970), cerebral asymmetry (Inglis, 1965), clinical investigations of the locus and extent of brain damage (Milner, Taylor, \& Sperry, 1968), and the screening of those with possible problems in reading and speech (Curry \& Gregory, 1969). It has also been used extensively in the experimental investigation of speech perception (Studdert-Kennedy \& Shankweiler, 1970), and short-term memory (Yntema \& Trask, 1963).

Difficulties in the preparation of adequately aligned dichotic tapes have often restricted experimentation. Trial-and-error recording, testing on an oscilloscope for alignment, and recording usually with splicing, is most frequently used, but is very time consuming. Computer control of alignment has been employed by, e.g., Treisman and Riley (1969), but this is expensive and demanding, and requires a number of peripherals. So also does the minicomputer technique of Knight and Kantowitz (1973).

A device ${ }^{1}$ is described below which controls three completely unmodified tape recorders (TRs) (see Figure 1). I he only constraints on the latter are that they have an accurate and unvarying system for remote control start and stop, and that they can be set to achieve delay-

This work was supported by an award from the Australian Research Grant Committee to J. L. Bradshew. We acknowledge the work of Dr. Terry Myer of the Edinburgh group who developed DITMA (unpublished). The device reported here is, however, considerably more flexible and requires no tape recorder modifications. Address reprint requests to Terry Vincent. Department of Psychology. Monash University, Clayton, Australia 3168 . ed auditory feedback (DAF). Revox Model A77 is ideal for this purpose, but other makes might be satisfactory. Essentially, the device locates the onset of each recorded speech burst before it reaches the TR read-head, by by means of a prerecorded tone burst located at a constant distance $(350 \mathrm{msec})$ on another channel before the speech burst. The two TRs (each carrying one of the two tracks for subsequent dichotic realignment and rerecording), are thus independently stopped and aligned. They are then restarted in synchrony with the third machine which rerecords the two aligned tracks, step by step (see Figure 2). Adjust facilities permit exact synchroni-

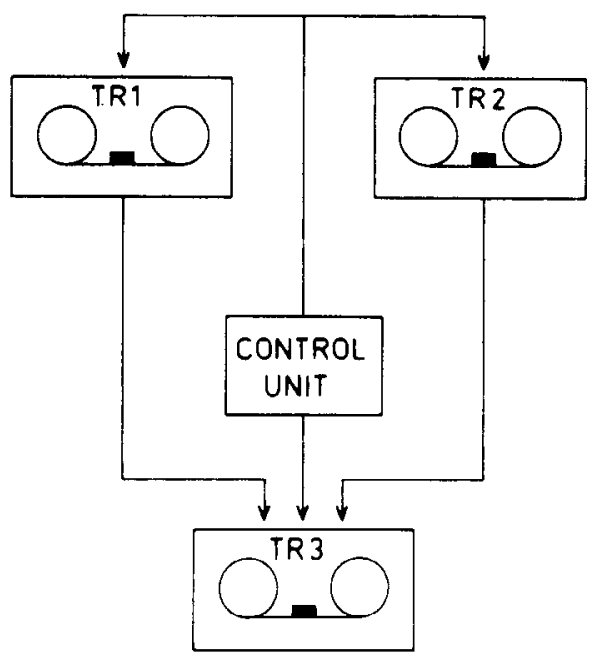

Figure 1. Manner in which dichotic tapes are automatically constructed. 


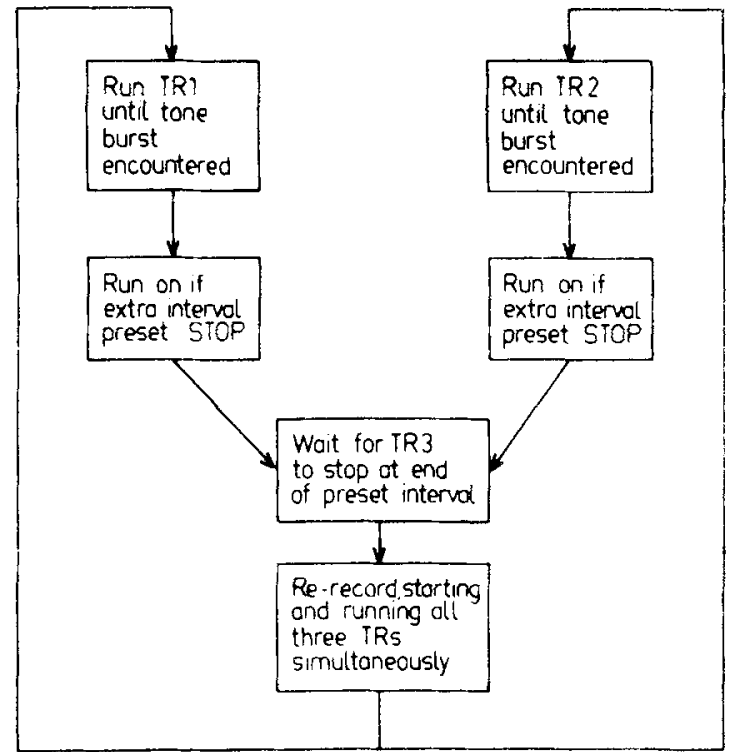

Figure 2. Block diagram of the sequence of events initiated by the control unit.

zation (see Moray \& O'Brien, 1967, for the need of this) should the two machines have variable start and stop lags, and also desynchronization, if desired, such as for an alternating left-right sequence. There is also a facility for a variable interpair interval, from $300 \mathrm{msec}$ to $10 \mathrm{sec}$.

The placing of a tone burst on tape (on a different channel), prior to the speech signal which is to be a. ligned to its pair member, is achieved in the following manner. A tone burst $(50 \mathrm{msec})$ initiated by a Schmitt trigger is placed immediately on Channel 2 when a word is spoken, but the latter is delayed by first being played through another TR which has been set to the DAF setting. Thus, the alignment tone (Channel 2) always precedes each speech signal (Channel 1) by a constant amount ( $350 \mathrm{msec})$. To compensate for any pauses or silent periods within a word, a $500-\mathrm{msec}$ delay is incorporated into the tone burst generator. This allows silent periods of up to $500 \mathrm{msec}$, and the next tone burst will not be generated until this period has timed out.

It is possible to record each channel separately, in one's own time, the only constraint being that successive items are not initially recorded at a rate faster than approximately $2 / \mathrm{sec}$. There is no lower limit, and the tape of aligned stimuli can, of course, contain pairs sequenced at a rate of from $2 / \mathrm{sec}$ to 1 every $10 \mathrm{sec}$. Two tapes, corresponding to the two channels are prepared in this way. played back and synchronized by the control device, and rerecorded on the third TR at the desired intervals. Exact onset alignment (or misalignment) settings can be set up by testing through a two-channel storage oscilioscope. Synchronized rerecording can then be left to continue automatically.

\section{THE CONTROL UNIT}

The control unit (see Figure 3) consists of three circuits controlling TRs 1.2, and 3. A common start pulse triggers each circuit causing TR3 to start. After preset time intervals (to correct for differences in start and stop characteristics in the two TRs), TRs 1 and 2 will then also be started.

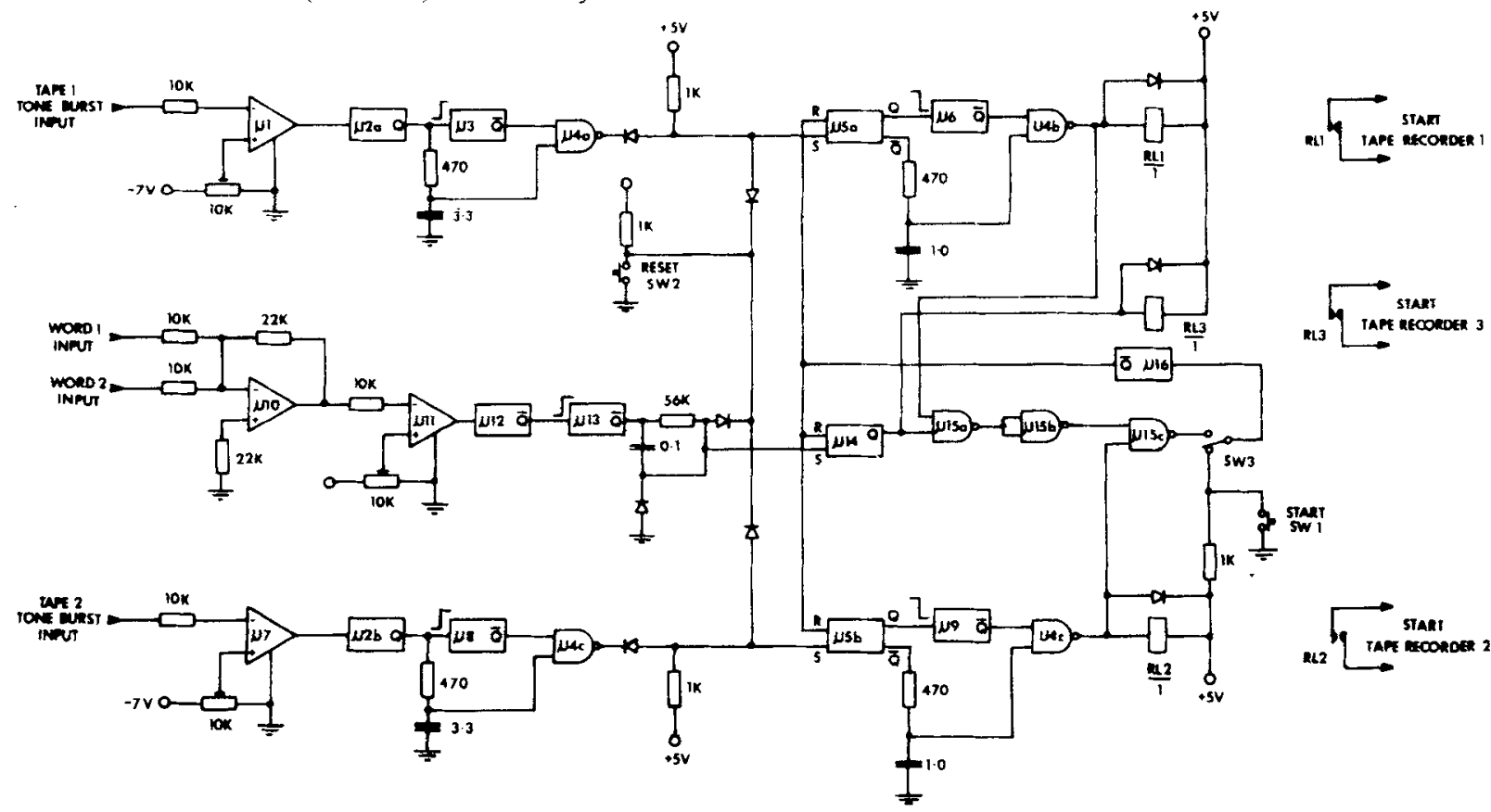

Figure 3. Circuit diagram of control unit. 
A word prerecorded on Channel 1 of TR1 is rerecorded onto Channel 1 of TR3. Simultaneously a word prerecorded on Channel 1 of TR2 is rerecorded onto Channel 2 of TR3. Tape Recorders 1 and 2 continue to run until a registration tone burst of $50-\mathrm{msec}$ duration is encountered on Channel 2 of TRs 1 and 2, which causes the two TRs to switch off. These two tone bursts are each recorded $350 \mathrm{msec}$ before onset of the two subsequent words to be aligned. In this way, TRs 1 and 2 are realigned with respect to one another, both being stopped at the same time interval before the onset of the next word to be rerecorded.

A list of components for the control unit is presented in Table 1.

\section{CIRCUIT OPERATION}

The start cycle is initiated either manually by means of the Start Switch SW1, or automatically by a pulse being generated when all 3 TRs have completed their cycles. The automatic/manual modes of operation are selected by Switch SW3.

When the start cycle is initiated, the output of the 50msec monostable Multivibrator $\mu 16$ changes state. This causes the outputs of the D-type Multivibrators $\mu 5 \mathrm{a}$, $\mu 5 \mathrm{~b}$, and $\mu 14$ to change state. Relay RL3 is energized by $\mu 14$ and through its contact TR3 will commence to record. Simultaneously monostable Multivibrator $\mu 6$ is triggered by $\mu 5 \mathrm{a}$ and monostable Multivibrator $\mu \overline{9}$ is triggered by $\mu 5 \mathrm{~b}$. Tape Recorder 1 will be delayed from starting with respect to TR3 by the time interval set by $\mu 6$. Similarly TR 2 will be delayed by the time interval set by $\mu 9$. Compensation for any time required for TR3 tape bounce or the difference in starting and stopping times of TR1 and TR2 can thus be controlled by adjusting the time durations for which $\mu 6$ and $\mu 9$ remain triggered. These time intervals, adjustable from $1 \mathrm{msec}$ to $1000 \mathrm{msec}$, are set by timing Resistors VR1 and VR2.

When $\mu 6$ times out, it enables the NAND Gate $\mu 4 \mathrm{~b}$ which energizes Relay RL1, and through its contacts TR1 will start. After $\mu 9$ has timed out, it enables NAND Gate $\mu 4 c$, which energizes Relay RL2 and TR2 starts. The words prerecorded on Channel 1 of TR1 and TR2 are rerecorded on Channels 1 and 2 of TR3.

Outputs 1 and 2 of TR 3 are added together by operational Amplifier 10 and then presented to the input to Comparator $\mu 11$. The threshold level of Comparator $\mu 11$ is set by a $10 \mathrm{~K} \Omega$ potentiometer which is adjusted so that the output will change state only when a word is present. This level is set above any TR output noise to avoid false resetting. When the words are being recorded by TR3, the output of $\mu 11$ changes state; this causes the retriggerable Multivibrator $\mu 12$ to trigger and remain in the triggered state for $100 \mathrm{msec}$ after the completion of
Table 1

List of Components for Control Unit

\begin{tabular}{lc}
\hline Integrated Circuits & \\
$\mu 1, \mu 7, \mu 11, \mu 18$ & LM 710 \\
$\mu 2, \mu 12, \mu 19$ & 9602 \\
$\mu 3, \mu 6, \mu 8, \mu 9, \mu 16, \mu 20, \mu 21$ & 74121 \\
$\mu 4, \mu 15$ & 7400 \\
$\mu 5, \mu 14$ & 7474 \\
$\mu 10, \mu 17$ & LM 741
\end{tabular}

Diodes

All diodes

IN 914

Relays 1, 2, 3

Grigsby-Barton GM 832A-1

$5 \mathrm{~V}, 10 \mathrm{~mA}$

Potentiometers

VR1, VR2, VR3

TEN TURN

HELIPOT $20 \mathrm{~K} \Omega$

both words, at which time it triggers monostable Multivibrator $\mu 13$. The time duration for which $\mu 13$ remains triggered is variable from $1 \mathrm{msec}$ to $10 \mathrm{sec}$ and is adjusted by means of Timing Resistor VR3. This time duration represents the time between words recorded on TR3. When $\mu 13$ times out it resets $\mu 15$ which deenergizes Relay RL3 and stops TR3. The Channel 2 output of TR1 is connected to the input of Comparator $\mu 1$. Tape Recorder 1 continues to run on after its word has been recorded until a tone burst recorded on Channel 2 of TR1 causes the output of $\mu 1$ to change state, triggering the 5-msec retriggerable Multivibrator $\mu 2 \mathrm{a}$ which in turn triggers 50 -msec Multivibrator $\mu 3$. As the length of the tone burst is $50 \mathrm{msec}, \mu 2 \mathrm{a}$ remains triggered for $55 \mathrm{msec}$. After $\mu 3$ times out it enables NAND Gate $\mu 4 \mathrm{a}$. Since $\mu 3$ is still triggered, $\mu 4 \mathrm{a}$ 's output changes state, resetting $\mu 5 \mathrm{a}$, which disables NAND Gate $\mu 4 \mathrm{~b}$ and thus de-energizes Relay RL1. Tape Recorder 1 now stops approximately $300 \mathrm{msec}$ before the start of the next word. The circuit comprising $\mu 2 \mathrm{a} . \mu 3$, and $\mu 4 \mathrm{a}$ is to prevent noise on the tape from stopping TR1 falsely.

Channel 2 of TR2 is connected to the imput of Comparator $\mu 7$. Tape Recorder 2 continues to run after its word has been recorded until the tone burst recorded on Channel 2 of TR2 causes the output of $\mu 7$ to change state. This triggers the 5 -msec retriggerable monostable Multivibrator $\mu 2 \mathrm{~b}$, which in turn triggers $50-\mathrm{msec}$ Multivibrator $\mu 8$. As $\mu 2 \mathrm{~b}$ is triggered for $55 \mathrm{msec}$, the output of NAND Gate $\mu 4 c$ changes state when $\mu 8$ has timed out. This resets $\mu 5 \mathrm{~b}$ which disables NAND Gate $\mu 4 \mathrm{c}$, de-energizing the Relay RL2. Tape Recorder 2 now stops approximatedly $300 \mathrm{msec}$ before the start of the next word, with the result that both TRs are now realigned. The threshold levels of the Comparators $\mu 1$ and $\mu 7$ are set by $10 \mathrm{~K} \Omega$ potentiometers which are adjusted so that the output will only change state when the tone burst is presented. This level is set above any TR output noise to avoid false resetting. 


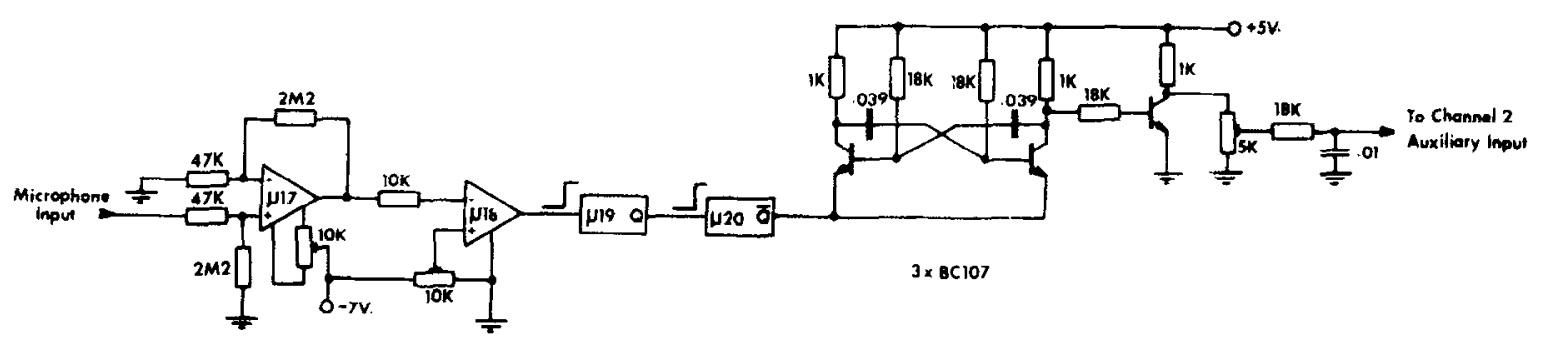

Figure 4. Circuit diagram of tone-burst generator.

\section{AUTOMATIC OPERATION}

The outputs of $\mu 4 \mathrm{~b}$ and $\mu 14$ are connected to the inputs of NAND Gate $\mu 15 \mathrm{a}$. When $\mu 4 \mathrm{~b}$ is disabled and $\mu 14$ is reset, this causes the output $\mu 15 \mathrm{a}$ to change state. This state is then negated by NAND Gate $\mu 15 b$ enabling NAND Gate $\mu 15 \mathrm{c}$. When $\mu 4 \mathrm{~d}$ is disabled it causes the output of $\mu 15 \mathrm{c}$ to change state and trigger $2 \mathrm{sec}$ monostable Multivibrator $\mu 21$. When $\mu 21$ has timed out it causes the 50-msec monostable Multivibrator $\mu 16$ to trigger via the contacts of Switch SW3, thus restarting the cycle.

\section{RESET SWITCH SW2}

When the control unit is initially switched on, all 'd' type multivibrators can be reset by depressing SW2. If the cycle is interrupted, the " $D$ " type multivibrators will need to be reset by depressing SW2.

\section{TONE BURST GENERATOR}

A microphone is connected to the input of a tape recorder (TRa) and also the input of an operational amplifier $\mu 17$. The output of $\mu 17$ is connected to a comparator $\mu 18$. When a word is spoken into the microphone, it causes the output of $\mu 18$ to change state. This triggers the $500-\mathrm{msec}$ retriggerable monostable multivibrator $\mu 19$, causing in turn the $50-\mathrm{msec}$ monostable multivibrator $\mu 20$ to trigger. In triggering, $\mu 20$ switches on a $1 \mathrm{KHz}$ oscillator which remains on for the $50 \mathrm{msec}$ for which $\mu 20$ is set. The output of the oscillator is connected to the Channel 2 input of $\mathrm{TRb}$ and thus a 50-msec tone burst is recorded on Channel 2 of $\mathrm{TRb}$. The output of TRa is connected to the Channel 1 input of $\mathrm{TRb}$. Tape recorder " $\mathrm{a}$ " is set to NAB so that the output is delayed with respect to the input by 350 msec, when a speed of $33 / 4$ is selected. The word now recorded on $\mathrm{TRb}$ is also delayed by $350 \mathrm{msec}$ with respect to the tone recorded on Channel 2.

\section{ALIGNMENT ACCURACY AND CALIBRATION}

The Revox A77 has special features to ensure accuracy of tape speed. Other recorders lacking this feedback system may prove considerably less desirable. However, no two tape recorders have exactly the same start and stop characteristics. Consequently, it is important to determine the necessary control unit settings for onset (or midpoint or offset) alignment, as required, and to determine the system's moment-to-moment accuracy. We used the following method.

When the system described above was set to deliver synchronized tapes, moment-to-moment alignment accuracy was determined in the following manner, Pure tone bursts of $2 \mathrm{KHz}$ and $100 \mathrm{msec}$ duration (corresponding roughly to the middle of speech frequencies, and the duration of the shortest spoken syllable) were recorded. Settings were adjusted on the control unit so that over 20 consecutive trials there was zero average misalignment error, as indicated by successive measurement of a two-channel oscilloscope. With these settings, another 40 test trials were run, and for each individual pair the extent was measured to which Channel 1 and Channel 2 led the other channel. Under these conditions, the average misalignment (now without regard to which channel was leading) was $1.2 \mathrm{msec}$, standard deviation 1.1 .

Exactly the same procedure was adopted for two types of speech signal. The words "bag," and "hack" were chosen. The former starts and terminates with voiced plosives, and the latter starts and terminates with unvoiced consonants, respectively, aspirate and stop. With control unit settings the same as with the pure tones, average misalignment values (standard deviations in brackets following) are given for the following dichotic pairs: bag/bag $3.3 \mathrm{msec}$ (4.5); bag/hack 2.4 $\operatorname{msec}(4.3)$.

Exact onset alignment of words differing greatly in onset characteristics can be obtained as follows. Average alignment accuracy should be obtained by setting the control unit and using pure tones, as above. The positions should be noted. A dichotic tape of the desired verbal material should then be prepared in the usual way, with the above settings. This tape should then be run through a two-channel oscilloscope, and onset misalignments noted for each word pair. Then from the original two tapes recorded prior to alignment, another dichotically-aligned tape should be prepared, this time with manual control of operations. Prior to each word pair being rerecorded, the appropriate lead/lag adjustment should be made on the control unit in accordance with the previously noted figures for that word pair. In this way onset (or midpoint or offset) alignment can be 
obtained as desired, to an accuracy of a few msec.

\section{TAPE BOUNCE AND FLUTTER}

It may occasionaly be found that the $350-\mathrm{msec}$ gap between tone burst and speech signal is too short to accommodate stopping and starting the tape recorder. It will be apparent in flutter during the early part of one or both of the aligned speech signals. In may be overcome by increasing to $700 \mathrm{msec}$ the gap between tone and speech signals. The tape recorder used to delay the speech signal (TRa) will continue to be set to $33 / 4$, but $\mathrm{TRb}$ (which received two signals) will be set to $71 / 2$, thus doubling the tape distance between the two signals. On recording, all three tape recorders (TR1, TR2, TR3) are set to $33 / 4$, to take advantage of the doubled tape distance. However, the rerecorded dichotically aligned tape must subsequently be played at $71 / 2$, to preserve the speech frequencies. The interpair intervals are also halved, and appropriate compensation must be set on the control unit for TR3 run on, i.e., a setting equal to double the desired value must be employed. It does, however, have a further advantage; any moment-to-moment alignment discrepancies are also halved.

\section{MAXIMUM PRESENTATION RATE}

Using the above system, and $100-\mathrm{msec}$ pure tone bursts, it was found that the maximum possible sequencing rate of dichotically aligned pairs was 2 every $1.3 \mathrm{sec}$. Obviously the longer the spoken material, the lower the possible repetition rate. It is not possible, of course, to control the word durations, as can a minicomputer system, though there is no reason why standard speechcompression techniques if available should not be used with the system described above, to achieve the same result.

\section{INTENSITY CONTROL}

A perennial methodological problem in the construction of dichotic tapes is the equalization of intensity, particularly across channels, where different words occur. It is desirable that a trained speaker be employed who can maintain a constant voice level. Mouth-to-microphone distance and orientation should be kept constant throughout recording sessions. The recording-level controls, balance, and playback intensities should be set, using both Vu meters and precision sound level meters, such as the Bruel and Kjaer Type 2203. The latter in particular should be used to calibrate test tones, or the same word repeated, on both channels of the final tape. However, it should be noted that both speech channels are initially recorded on the same machines and on the same channels, though at different times. This will tend to reduce any possible variability.

\section{REFERENCES}

Broadbent, D. E. The role of auditory localization in attention and memory span. Journal of Experimental Psychology, $1954,47,191-196$.

Curry, F. K. W., \& Gregory, H. H. The performance of stutterers on dichotic listening tasks thought to reflect cerebral dominance. Journal of Speech and Hearing Research, 1969, 12 . $73-82$

Inglis, J. Dichotic listening and cerebral dominance. Acta Otolaryngologica, 1965, 60, 231-238.

Knight, J. L., \& Kantowitz, B. H. A minicomputer method for generating dichotic word pairs. Behavior Research Methods \& Instrumentation, 1973, 5, 231-234.

Lew1s, J. L. Semantic processing of unattended message using dichotic listening. Journal of Experimental Psychology, $1970,85,225-228$.

Milner, B., Taylor, L., \& Sperry, R. W. Lateralized suppression of dichotically-presented digits after commissural section in man. Science, 1968, 161, 184-185.

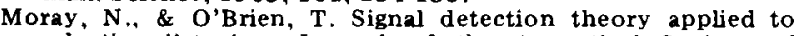
selective listening. Journal of the Acoustical Society of America, 1967, 42, 765-772.

Studdert-Kennedy, $M_{.,}$\& Shank weiler, D. Hemispheric specialization for speech perception. Journal of the Acoustical Society of America, 1970, 48, 579-594.

Treisman, A. M., \& Riley, J. G. Is selective attention selective perception or selective response? A further test. Journal of Experimental Psychology, 1969, 79, 27-31.

Yntema, D. B., \& Trask, F. P. Recall as a search process. Journal of Verbal Learning and Verbal Behavior, 1963, 2, 65-74.

1. This device is the subject of an application for a patent on file in Australia. 